\title{
CONTRIBUCIÓN DE LAS TI EN LA PROBLEMÁTICA MEDIO AMBIENTAL Y SU INCIDENCIA EN INSTITUCIONES DE EDUCACIÓN SUPERIOR
}

\section{CONTRIBUTION OF IT IN THE ENVIRONMENTAL PROBLEMS AND ITS IMPACT ON HIGHER EDUCATION INSTITUTIONS}

\author{
Freddy Anibal Marcillo Merino ${ }^{1}$ \\ Diana Victoria Marcillo Parrales ${ }^{2}$ \\ Xavier Enrique Soledispa Rodríguez ${ }^{3}$ \\ Christian Ruperto Caicedo Plúa ${ }^{4}$
}

1. Doctor PhD en Ciencias Pedagógicas, Magister en Gerencia de Proyectos Educativos y Sociales, Ingeniero Agrónomo, Diplomado en autoevaluación y acreditación Universitaria por la Universidad Aconcagua de Chile, Licenciado en Ciencias de la Educación, Docente Titular de la Universidad Estatal del Sur de Manabí, Carrera Administración de Empresas Agropecuarias. Email: fmarcillo2010@yahoo.es

2. Magister en auditoria y contabilidad en la Universidad Estatal del Sur de Manabí, Economista, Docente principal de la Carrera de Administración de Empresas Agropecuarias, en proceso de Doctorado en la Universidad Pinar del Rio de la República de Cuba, Email: divi24marpa@yahoo.com

3. Economista, Docente Titular de la Universidad Estatal del Sur de Manabí, Carrera Gestión Empresarial, Email: xsoledispa@hotmail.com

4. Magister en Gerencia Educativa e Investigación, Ingeniero en Computación y Redes, Docente Titular Investigador Auxiliar 1 de la Universidad Estatal del Sur de Manabí, Carrera Ingeniería en Computación y Redes. Email: christiancaicedoplua@hotmail.com

\section{Citación sugerida:}

Marcillo Merino, F.A., Marcillo Parrales, D.V., Soledispa Rodríguez, X.E., y Caicedo Plúa, C.R. (2016). Contribución de las $\mathrm{TI}$ en la problemática medio ambiental y su incidencia en instituciones de educación superior. 3C Empresa, investigación y pensamiento crítico, 5(4), 41-57. DOI: <http://dx.doi.org/10.17993/3cemp.2016.050428.41-57/>. 


\section{RESUMEN}

La presente investigación cuali - cuantitativa tiene como objetivo identificar los problemas ambientales ya que son alteraciones organizadas por actividades humanas o condiciones naturales del medio, cuyo objeto es mejorar la calidad de vida de la sociedad, por lo cual se realizó un análisis sobre la incidencia de la cultura ambiental sostenible a la comunidad de la UNESUM a través de las TI, para que no depositen basura al suelo y que lo ubiquen en su lugar, es decir reciclar los desechos, por medio de la identificación de estrategias para atacar causas y efectos negativos que producen la Contaminación. Los instrumentos y técnicas utilizados permitieron identificar la relación entre las variables, entre las cueles se tiene a encuesta y observación lo que permitió identificar las condiciones y hechos, aplicados a los objetivos del presente artículo.

\section{ABSTRACT}

This qualitative research - quantitative aims to identify environmental problems and that are organized by human activities or natural environmental conditions changes, aimed at improving the quality of life of society, for which an analysis of the incidence was carried out environmental culture sustainable community of UNESUM through iT, not to place trash on the ground and that locate in place, ie recycle waste, by identifying strategies to tackle causes and negative effects Pollution produced. The tools and techniques used allowed to identify the relationship between the variables, between you strain it has to survey and observation allowing to identify the conditions and events, applied to the objectives of this article.

\section{PALABRAS CLAVE}

TI, medio ambiente, educación superior, cultura ambiental, sostenible.

\section{KEY WORDS}

$\mathrm{TI}$, the environment, higher education, environmental culture, sustainable. 


\section{INTRODUCCION}

"El hombre debe aprender que el ambiente no es algo que pueda manejar según su voluntad, sino que él debe integrarse para tener una vida mejor, y un primer paso importante para perfeccionar el habitad............el objeto principal sería lograr que el hombre cambie de actitud interna hacia su ambiente respetando sus valores y derechos" Autores.

A inicios del siglo XXI aparece el término medio ambiente relacionado con las tecnologías de la información y comunicación (TIC), dentro de esta perspectiva se determina el cuidado y respeto al mismo, es importante tener en cuenta que una óptima educación ambiental debe vincular la academia, sociedad, entidades públicas y privadas permitiendo así el fortalecimiento del adelanto local con buenas prácticas ambientales en diversas aristas de desarrollo (Cabero, 2005).

El desarrollo de las nuevas tecnologías en los actuales momentos avanza de manera acelerada ya que vivimos en una sociedad consumista, a cada hora se producen miles de dispositivos electrónicos cuyo objeto es facilitar las diferentes tareas de los usuarios sin tener en cuenta las secuelas ambientales y sociales que esto conlleva, creando un problema medio ambiental por los residuos tecnológicos (Valdiviezo, 2012).

Desde esta perspectiva es importante recalcar que los individuos deben evolucionar su mapa mental actual en donde la formación en valores permita fortalecer el buen vivir de la humanidad, ligado a la formación mínima de aptitudes y capacidades medio ambientales. Por lo tanto, el uso de las TIC se ha convertido en un elemento de perfeccionamiento y mejora de las sociedades, logrando llegar a todos los sectores y áreas de conocimiento.

De acuerdo a las teorías medio ambientales y a la perspectiva pragmática se puede definir al medio ambiente como un ente directo que mejora la calidad de vida y preservación del patrimonio en todas sus formas en donde la (solidaridad, convivencia, diversidad, atención, cuidado y la participación de los individuos) forman un rol muy importante por medio de una buena gestión ambiental (Cabero J. , 2010).

Desde la perspectiva educativa, el medio ambiente como campo de formación del individuo es trascendental ya que a través de un programa de estudio, planes analíticos mediante resultados o competencias se pueden formar personas más conscientes de la problemática medio ambiental actual. De aquí que las IES tienen un papel trascendental, pues son el eje motriz para el cambio de paradigma dentro del entorno social - cultural y político (Sureda, 2001).

Desde esta perspectiva de sustentabilidad, el relieve y comportamiento de la naturaleza, permiten realizar una propuesta para entender y solucionar la Insuficiencia en el proceso docente educativo para lograr la formación de una cultura ambiental sustentable, cuyo objeto pretende fortalecer el proceso docente educativo en la concientización de los problemas ambientales (Caicedo, Ch. Rodriguez, A. Caicedo, F. Acuña, R. Delgado, G., 2015). Dentro del campo del presente trabajo de investigación este determinado por el proceso de formación de una cultura ambiental sustentable en la asignatura Ecología y Educación 
Ambiental, como materia integradora. Como solución del hecho científico se tiene: Instrumentar una estrategia didáctica de formación de una cultura ambiental sustentable para el nivel superior para formar progresivamente dicha cultura en los alumnos de este nivel y demás involucrados.

\subsection{LAS TIC EN EL PROCESO DE FORMACIÓN DEL INDIVIDUO}

El avance en el desarrollo de la tecnología actualmente ha propiciado lo que se denomina "Sociedad de la Información" en donde se presenta como eje motriz la "información" ya que esta es el eje transversal de desarrollo de la ciencia y la academia de la nueva sociedad de conocimiento (Arroyo, Valera, 2004).

Dentro de la catedra es de real importancia tener en cuenta los recursos didácticos (audiovisuales e informáticos) que utiliza el catedrático para fortalecer los contenidos teóricos a los educandos. El aprendizaje dentro del aula es en base a estrategias y técnicas que empleemos para alcanzar los objetivos propuestos, desde esta perspectiva el catedrático es el componente más significativo que permite concatenar el desarrollo de la enseñanza / aprendizaje (Adell, 1996).

El educando lo podemos definir como un receptor activo y consiente de la información que le es mostrada de tal manera que él a través de sus aptitudes y destrezas adquiridas determina diversos efectos cognitivos específicos que influyen en el aspecto psicomotriz y afectivo del individuo en el medio (Sigea, 2016).

El medio lo podemos definir como una serie de mecanismos internos y externos que propician aprendizajes generales y específicos a través de la experiencia. Por lo tanto, la conjunción entre el docente, educando, medio y la catedra permiten generar cambios significativos en la formación del individuo.

Las TIC pueden fortalecer los procesos de formación conceptual y técnica en el área de educación ambiental en donde el estudiante y docente son protagonistas de cambios significativos en la motivación y transmisión de información en la comunidad. De acuerdo a este criterio se debe realizar una selección concreta de contenidos y objetivos que se desean alcanzar y trasmitir teniendo en cuenta la edad, nivel socio - cultural y educativo en el medio, ya que las diferencias epistemológicas entre estudiantes pueden condicionar los resultados (Castells, 2001).

Las herramientas TIC más utilizadas actualmente por los docentes para el desarrollo de la catedra son: multimedia, video conferencias, internet, streaming, entre otros. Elementos que adquieren relevancia en el desarrollo de aprendizajes cooperativos y colaborativos dentro del aula mediante el uso de nuevas tecnologías (Vizcarro, C. y León, J. A., 2010).

La influencia de la tecnología sobre la sociedad marca un hito trascendental, ya que nos lleva a descubrir nuevos escenarios y proyectos que deben llevarnos a través de la investigación y el análisis de sus efectos a tomar enfoques que marquen la dirección a seguir para desarrollar la sociedad que deseamos construir (Crook, 2009). 


\subsection{RELACIÓN ENTRE EDUCACIÓN Y MEDIO AMBIENTE}

Al medio ambiente se lo considera como un medio físico con elementos abióticos de la naturaleza, ambiente, ecosistema, habitad o entorno. Según la UNESCO (1977) es "un conjunto de sistemas naturales y sociales en que viven el hombre y los demás organismos y de donde obtienen su subsistencia".

Los problemas ambientales son de complejidad muy diversa en donde el ser humano cumple un rol fundamental. La formación desempeña una tarea esencial ya que la presencia del sector educativo permitirá fortalecer conocimientos y valores para el cuidado ambiental. Para develar la brecha epistemológica (Cañal, Porlán y García, 1981) recogen las inquietudes de la época, es decir, acerca de la Ecología y la Teoría de Sistemas, y consideran que la educación ambiental debe tener un carácter preferentemente social que afecte las esferas políticas económicas y sociales en cuestión. Es casi como bosquejan los siguientes fines:

a) Lograr un cambio insondable en las organizaciones, en las formas de análisis y en la gestión de las cuestiones referentes al medio;

b) Lograr que en la planificación se tenga en cuenta prioritariamente los conocimientos que la ciencia Ambiental pueda aportar;

c) Conseguir el establecimiento de principios éticos; y

d) Instituir un tipo de educación en el que la metodología utilizada sea la del contacto directo con la realidad del entorno.

Desde otra perspectiva la propuesta de Smyth (1996) radica en ver la educación ambiental como un proceso que se estructura en etapas:

a) La concientización ambiental, que es el proceso de alerta a la gente sobre los factores que influencian su ambiente;

b) La alfabetización ambiental;

c) La responsabilidad ambiental, que reconoce el papel especial de la humanidad en la determinación y orientación del cambio, así como en la capacidad de evaluar entre diferentes opciones; $y$

d) La ciudadanía ambiental que es un concepto de membrecía participativa en el sistema.

De igual forma la propuesta de Leff (2015), expresa que la necesidad de fundamentar la educación en un saber ambiental, entendiéndolo como un plan de revisión y reconstrucción del mundo a través de estrategias conceptuales y políticas que partan de principios y fundamentos de una racionalidad ambiental que han sido desterrados y marginado por los paradigmas dominantes de la ciencia. La misma propone estrategias conceptuales, como lo es la interdisciplinariedad, la racionalidad ambiental y un dialogo conceptual en el cual participan, tanto los saberes provenientes de la ciencia, así como un conjunto de saberes sin pretensión científica que deben ser revalorizados para construir nuevas racionalidades. 
Analizando estos criterios de y siguiendo a Cañal, Porlán y García (1998) son más concretos en el sentido de bosquejar claramente, que el conocimiento proveniente de la ciencia de la ecología es la que debe regir toda la lógica y fin de la educación, con lo cual pretenden poder afectar la estructura política, económica y cultural de la sociedad en cuestión. Sin embargo, no reconocen que la ecología por sí sola es insuficiente y sigue finalmente una lógica positivista, sin poder ofrecer una alternativa de fondo al complejo problema de lo ambiental.

Por lo tanto, la educación ambiental es todo un proceso que tiene que pasar por etapas diferentes; y por el otro lado, haciendo una aproximación sistemática al problema, dándole un papel especial a las humanidades. Esta propuesta considera de manera más integral el factor social, sin restringirlo a una lógica ecologista (Calvo, Susana; Corraliza, José Antonio, 2002).

Por lo tanto, la educación ambiental es un excelente proceso que, favorablemente encaminado, proyectado, realizado y valorado en los distintos grupos y sectores de nuestras sociedades, puede renovar y estimular los procesos políticos, económicos, sociales y culturales de nuestro entorno para contribuir a minimizar la pobreza, el analfabetismo, la falta de educación, carencia de servicios de salud y otros problemas globales contemporáneos, incluyendo los problemas del medio ambiente a nivel local, regional y mundial.

\subsection{RECICLAJE COMO ALTERNATIVA DEL BUEN VIVIR}

El reciclaje favorece al desarrollo de la cultura ambientalista y al beneficio sostenible de los recursos naturales del entorno. Frosch (2001) acota que el objetivo del reciclaje está enmarcado en los siguientes aspectos fundamentales:

a) Tipos de desechos;

b) Forma de utilizarlos; $y$

c) Posibles compradores de materiales reciclados como (papel, plástico, aluminio y vidrio).

Arnau (2000) expresa algunos puntos importantes dentro de las ventajas que se tiene con el reciclaje entre los que se denotan los siguientes:

1. Reduce la contaminación y aumenta la subsistencia de los recursos naturales.

2. Beneficia la conservación de la energía porque se requiere menos energía para hacer los productos de materiales reciclados.

3. Impide los costos de disposición de desechos en los rellenos sanitarios.

4. Reduce el volumen de basura que va a los rellenos prolongando su tiempo de vida útil.

5. Genera empleos, puesto que se necesita una fuerza de trabajo laboral para recolectar los materiales aptos para el reciclaje y su clasificación. 
6. Genera ingresos económicos.

7. Provee a las industrias fuentes menos costosas de materiales y en términos de costos, traslada las ventajas a los consumidores quienes gastan menos en productos y empaquetamientos.

8. Provoca la disciplina social en el manejo de los desechos.

9. Estimula el respeto a la naturaleza.

10. Beneficia el ahorro de agua potable.

11. Reduce el impacto en los ecosistemas.

12. Origina la organización de las comunidades.

13. Reduce riesgos sanitarios.

Dentro de las políticas públicas en la República del Ecuador se tiene el Plan Nacional del Buen Vivir que en su Objetivo 7. Expresa de manera tácita "Garantizar los derechos de la naturaleza y promover la sostenibilidad ambiental territorial y global". En donde se implementa un marco normativo que garantiza los derechos de la naturaleza e instaura mecanismos intersectoriales, transversales e integrados, de prevención, control, sanción y restauración integral de daños y pasivos socio ambientales, asegurando las compensaciones respectivas y la no repetición de los daños o afectaciones (Desarrollo, 2016).

De igual manera dicho documento expresa lo siguiente: asegurar la promoción, la vigencia y la plena exigibilidad de los derechos de la naturaleza, Conocer, valorar, conservar y manejar sustentablemente el patrimonio natural y su biodiversidad terrestre, acuática continental, marina y costera, con el acceso justo y equitativo a sus beneficios, Consolidar la gestión sostenible de los bosques, enmarcada en el modelo de gobernanza forestal, Impulsar la generación de bioconocimiento como alternativa a la producción primario-exportadora, Garantizar la bioseguridad precautelando la salud de las personas, de otros seres vivos y de la naturaleza, Gestionar de manera sustentable y participativa el patrimonio hídrico, con enfoque de cuencas y caudales ecológicos para asegurar el derecho humano al agua, Promover la eficiencia y una mayor participación de energías renovables sostenibles como medida de prevención de la contaminación ambiental, Prevenir, controlar y mitigar la contaminación ambiental en los procesos de extracción, producción, consumo y postconsumo, Promover patrones de consumo conscientes, sostenibles y eficientes con criterio de suficiencia dentro de los límites del planeta, implementar medidas de mitigación y adaptación al cambio climático para reducir la vulnerabilidad económica y ambiental con énfasis en grupos de atención prioritaria, Promover la consolidación de la Iniciativa Yasuní$I T T$, y Fortalecer la gobernanza ambiental del régimen especial del Archipiélago de Galápagos y consolidar la planificación integral para la Amazonía. 


\section{METODOLOGÍA}

Este trabajo se desarrolló utilizando el método de la encuesta y la observación, lo que permitió identificar las condiciones y hechos, aplicados a los objetivos del presente artículo, como también las causas más comunes, para su posterior argumentación de los resultados obtenidos en la investigación y dar a conocer que aporte tiene esta ante el tema.

En la actualidad, los principales problemas ambientales de la institución se han visto influidos por una falta de conciencia y educación ambiental, que han traído como consecuencia en muchas ocasiones, su agravamiento. El desarrollo de estos elementos, inciden directamente en la manera de actuar del ser humano sobre el medio ambiente, no ha estado a la altura de otras obras colosales llevadas a cabo por este proceso gubernamental, y de ahí que constituya un factor esencial de trabajo a corto y mediano plazo, para lograr resultados positivos en la implementación de la política ambiental y una gestión eficiente en la identificación de los principales problemas ambientales de la Universidad.

\section{RESULTADOS Y DISCUSIÓN}

Resultado de las encuestas dirigida a los profesionales de las carreras de enfermería y administración de empresas agropecuarias de la universidad estatal del sur de Manabí, sobre la temática del medio ambiente, con una población de un total de 50 encuestados.

1. ¿Importancia que atribuye usted al cuidado del medio ambiente?

Tabla 2. Cuidado del medio ambiente.

\begin{tabular}{|c|c|c|}
\hline Alternativas & Frecuencia & Porcentajes \\
\hline Mucho & 40 & $80 \%$ \\
\hline Poco & 10 & $20 \%$ \\
\hline Nada & 0 & $0 \%$ \\
\hline TOTAL & 100 & $100 \%$ \\
\hline
\end{tabular}

Fuente: elaboración propia.

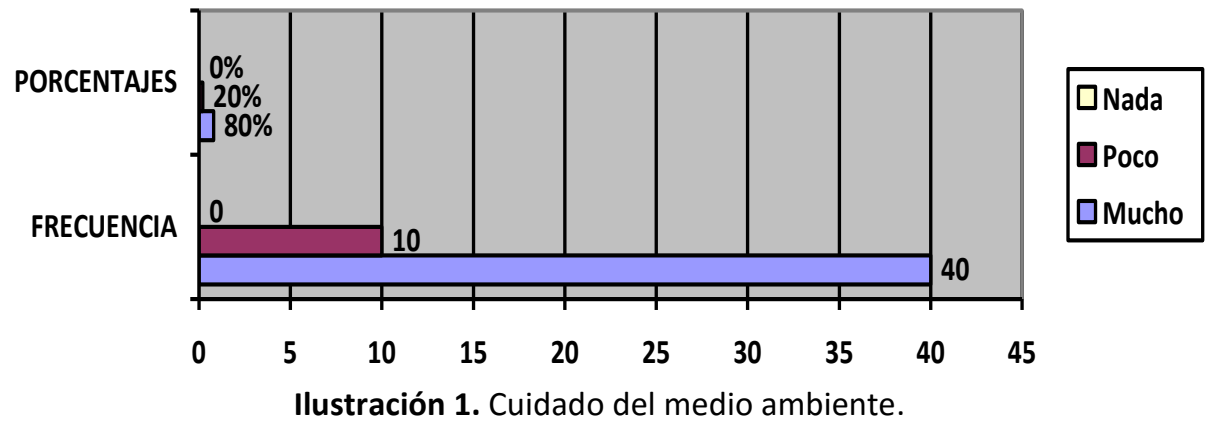

Fuente: trabajo de campo. Encuesta aplicada a Funcionarios de la UNESUM.

Elaborado por: investigadores. 
EL $80 \%$ indicó que tiene mucha importancia el cuidado del medio Ambiente y el $20 \%$ indico que existe poco cuidado con el medio ambiente, por lo cual resulta propicia buscar estrategias por medio de la inserción de nuevas tecnologías para el fortalecimiento del cuidado al medio ambiente.

2. ¿Qué importancia tiene para usted la cultura ambiental?

Tabla 3. Cultura ambiental.

\begin{tabular}{|c|c|c|}
\hline Alternativas & Frecuencia & Porcentajes \\
\hline Mucho & 40 & $80 \%$ \\
\hline Poco & 10 & $20 \%$ \\
\hline Nada & 0 & $0 \%$ \\
\hline TOTAL & 100 & $100 \%$ \\
\hline
\end{tabular}

Fuente: elaboración propia.

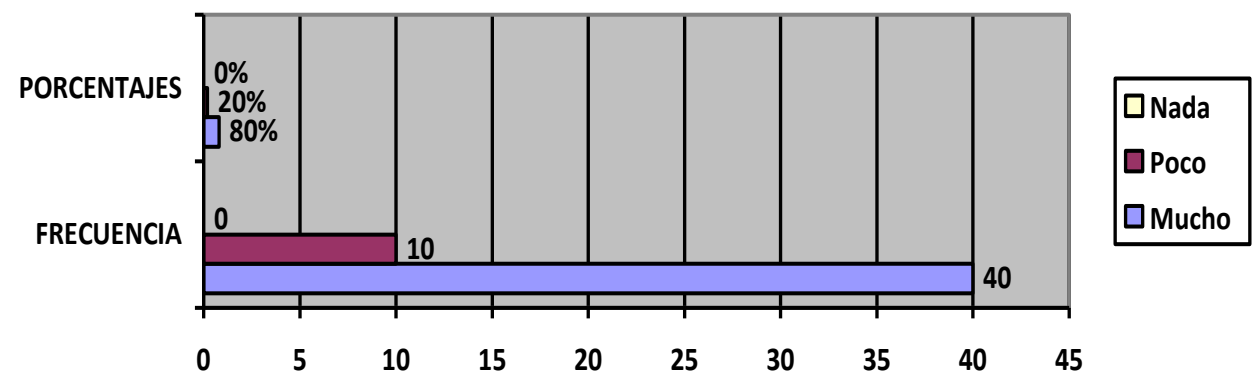

Ilustración 2. Cultura ambiental.

Fuente: trabajo de campo. Encuesta aplicada a Funcionarios de la UNESUM.

Elaborado por: investigadores.

EL $80 \%$ indicó que tiene mucha importancia la cultura ambiental y el $20 \%$ indicó que no tiene mucha importancia, por lo cual resulta propicia buscar estrategias por medio de la inserción de nuevas tecnologías para concientizar el cuidado del medio ambiente.

3. ¿Cómo valora en la parte de su formación profesional el cuidado del ambiente?

Tabla 4. Formación profesional al cuidado del medio ambiente.

\begin{tabular}{|c|c|c|}
\hline Alternativas & Frecuencia & Porcentajes \\
\hline Alto & 28 & $66 \%$ \\
\hline Medio & 20 & $30 \%$ \\
\hline Poco & 0 & $0 \%$ \\
\hline Nada & 2 & $4 \% \mathrm{~A}$ \\
\hline TOTAL & 100 & $100 \%$ \\
\hline
\end{tabular}

Fuente: elaboración propia. 


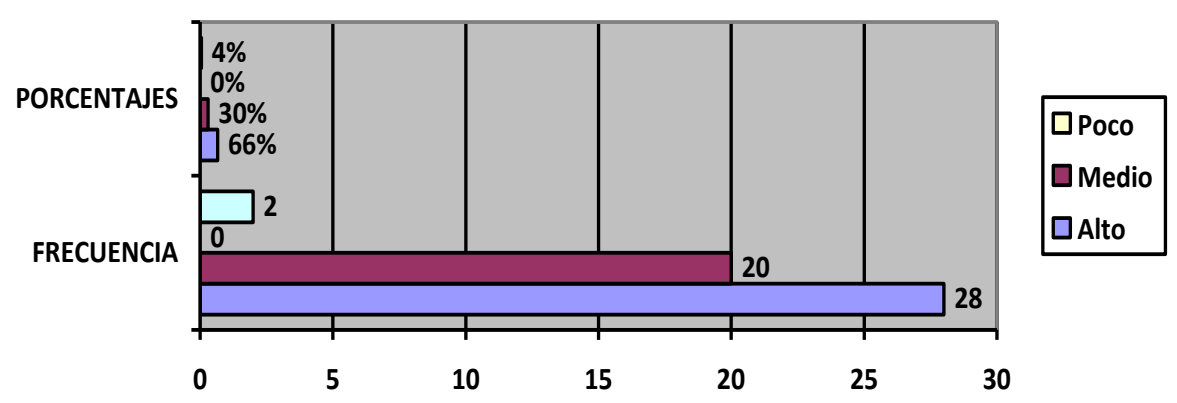

Ilustración 3. Formación profesional al cuidado del medio ambiente.

Fuente: Trabajo de campo. Encuesta aplicada a Funcionarios de la UNESUM.

Elaborado por: Investigadores.

El $66 \%$ valora alto en la parte de su formación profesional el cuidado del ambiente, el $30 \%$ medianamente y el $4 \%$ no valora, por lo tanto, desde la academia se debería de fortalecer la cultura ambiental como parte importante del currículo.

4. Como aprecia usted el respeto al ambiente en el entorno.

Tabla 5. Respeto al medio ambiente.

\begin{tabular}{|c|c|c|}
\hline Alternativas & Frecuencia & Porcentajes \\
\hline Mucho & 24 & $48 \%$ \\
\hline Poco & 24 & $48 \%$ \\
\hline Nada & 2 & $4 \% \mathrm{~A}$ \\
\hline TOTAL & 100 & $100 \%$ \\
\hline
\end{tabular}

Fuente: elaboración propia.

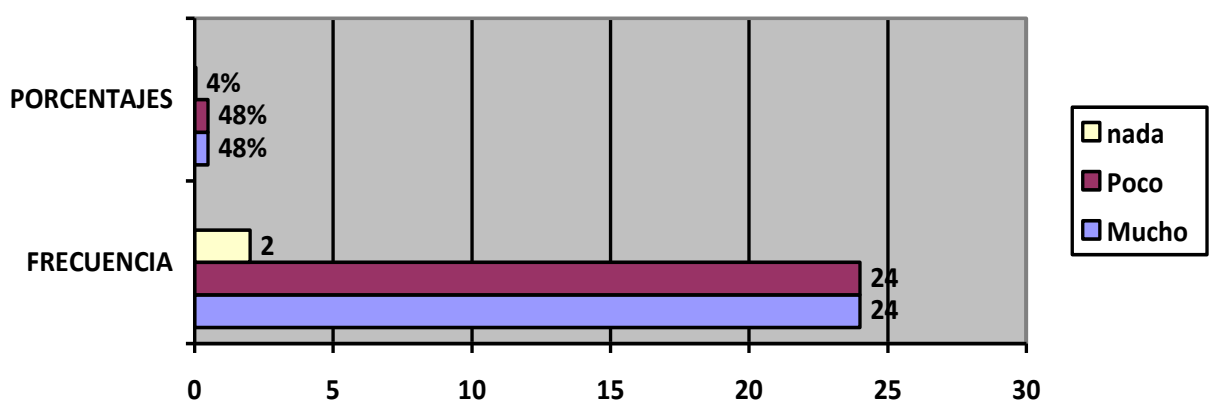

Ilustración 4. Respeto al medio ambiente.

Fuente: trabajo de campo. Encuesta aplicada a Funcionarios de la UNESUM.

Elaborado por: investigadores.

El 48\% indicó que mucho aprecia el cuidado ambiental en el entorno de la Universidad, y el $48 \%$ expresó que aprecia el cuidado ambiental en el entorno de la universidad y el $4 \%$ no aprecian el cuidado en el entorno de la Universidad. 
5. ¿Considera que la educación que recibe en la UNESUM sobre el cuidado del medio ambiente es?

Tabla 6. Educación que recibes en la UNESUM.

\begin{tabular}{|c|c|c|}
\hline Alternativas & Frecuencia & Porcentajes \\
\hline Excelente & 9 & $52 \%$ \\
\hline Muy Buena & 26 & $28 \%$ \\
\hline Buena & 14 & $18 \%$ \\
\hline Mala & 1 & $2 \%$ \\
\hline TOTAL & 100 & $100 \%$ \\
\hline
\end{tabular}

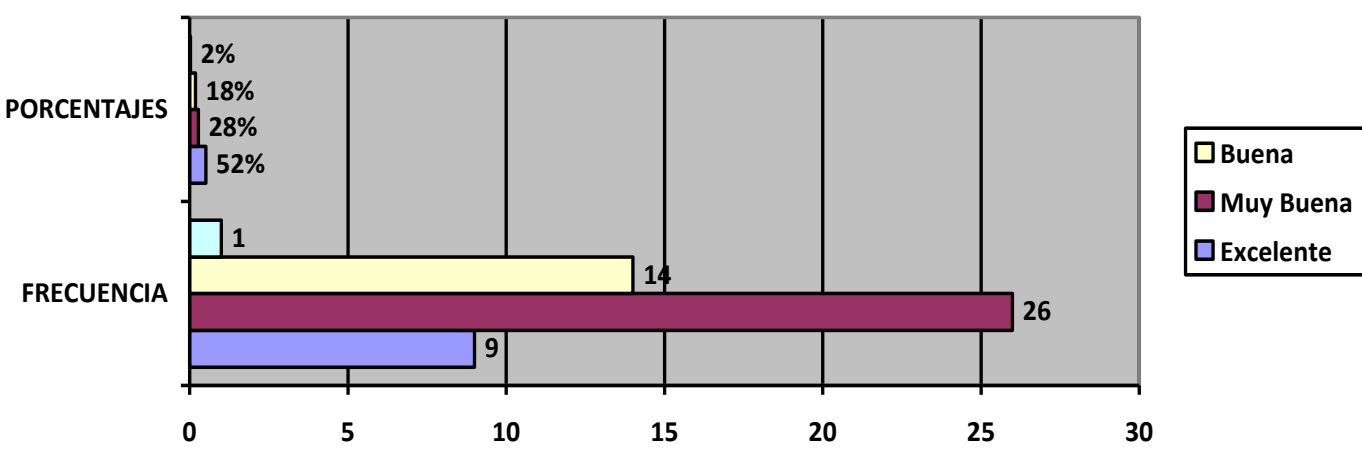

Ilustración 5. Educación que recibes en la UNESUM.

Fuente: trabajo de campo. Encuesta aplicada a Funcionarios de la UNESUM. Elaborado por: investigadores.

El 52\% indicaron que es Excelente la educación que reciben en la UNESUM, el 28\% que es buena, el $18 \%$ que es buena y el $2 \%$ expreso que es mala. Con lo cual, es necesaria la implementación de mecanismos que potencien la educación ambiental en la UNESUM.

6. ¿Cómo debe influir la universidad en la comunidad sobre el cuidado del MEDIO AMBIENTAL a través del uso de las TI?

Tabla 7. Cuidado medio ambiental a través de las TI.

\begin{tabular}{|c|c|c|}
\hline Alternativas & Frecuencia & Porcentajes \\
\hline Concientizándolos & 25 & $50 \%$ \\
\hline Informándolos & 9 & $26 \%$ \\
\hline Educándolos & 13 & $18 \%$ \\
\hline Nulo & 3 & $6 \%$ \\
\hline TOTAL & 100 & $100 \%$ \\
\hline
\end{tabular}

Fuente: elaboración propia. 


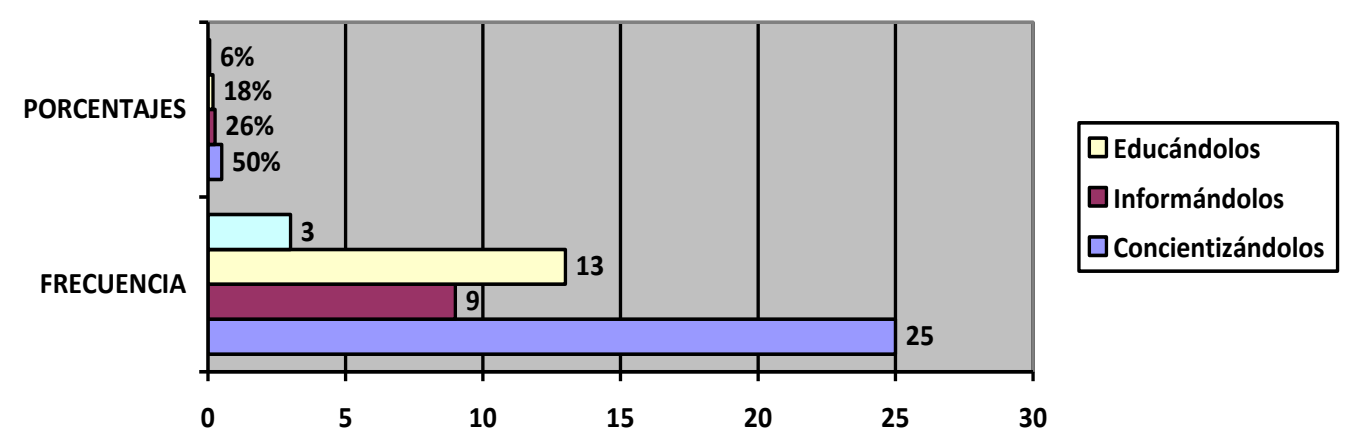

Ilustración 6. Cuidado medio ambiental a través de las TI.

Fuente: trabajo de campo. Encuesta aplicada a Funcionarios de la UNESUM.

Elaborado por: investigadores.

El 50\% por ciento de los encuestados indicaron que debe de concientizarse a la comunidad sobre el cuidado del medio ambiente, el $26 \%$ expreso que educándolos, el $18 \%$ informándole y el $6 \%$ no sugieren nada. Con lo cual, es primordial realizar un proceso vinculante para potenciar la cultura ambiental a través de las nuevas tecnologías.

Por lo tanto, el objeto de la educación ambiental es reintegrar circunstancias de interacción entre el hombre/hombre y hombre/naturaleza que definan perspectivas críticas e innovadoras y que estas contribuyan a la transformación positiva de la comunidad.

Desde esta conceptualización, la educación ambiental pretende que los usuarios asimilen la naturaleza compleja de lo que comprende el medio ambiente natural y el establecido por el hombre y obtengan una cultura encuadrada en valores, comportamientos, y las habilidades prácticas para ser partícipes responsables en la prevención y solución de los problemas ambientales.

De tal forma que a través de estrategias se permita concienciar a la comunidad Universitaria para el desarrollo de actividades que permitan concienciar sobre el cuidado del medio ambiente mediante el uso de las Tecnologías de la Información y Comunicación. 


\subsection{FUNDAMENTACIÓN DEL PROGRAMA DE G.A. UNESUM}

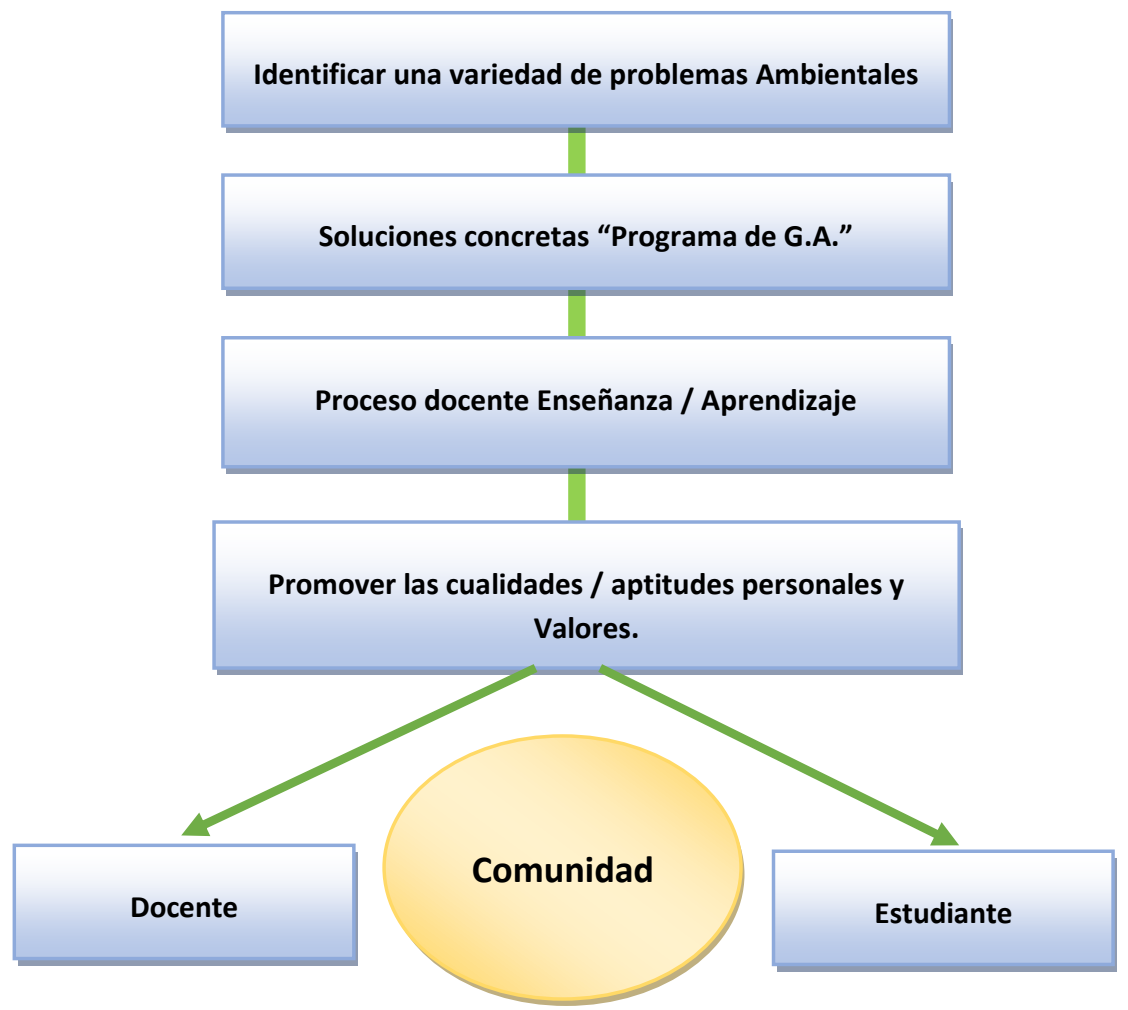

Gráfico 1: Fundamentos del programa de G.A. UNESUM.

Realizado por: autores.

Es importante tener en cuenta que para la aplicación de este modelo se deben desarrollar habilidades pedagógicas que conlleven a satisfacer necesidades de la comunidad.

Este programa se sustenta en principios fundamentales enmarcados en la normativa constitucional del Buen Vivir y que tienen relación con el reconocimiento al derecho del usuario a un condición de vida sana, sistematicidad, lo cognitivo, lo conductual y la transformación de actitudes, la unidad del Medio Ambiente natural, el desarrollo, el enfoque y carácter de multidisciplinariedad, interdisciplinariedad y transdisciplinariedad, el mejoramiento de la calidad de vida, creación de una cultura general integral, inclusión de la dimensión ambiental y el establecimiento de procesos de capacitación y educación permanente para los profesionales y estudiantes de la Universidad Estatal del Sur de Manabí a través de un programa de educación ambiental.

De esta forma, la aplicación de las TI hoy en día es esencial en todas las áreas de la sociedad, ya que por medio de ella se sistematizan procesos para mejorar la calidad de vida de las personas a través del cuidado del medio ambiente.

Por consiguiente, la estrategia incorporada al cuidado del medio ambiente y al uso de las nuevas tecnologías conlleva a tener una sociedad más concientizada, como legado para las futuras generaciones. Uno de los propósitos de esta investigación fue promover el cuidado y respeto por la naturaleza, además de vincular las nuevas tecnologías para beneficio social. 


\subsection{PROGRAMA DE EDUCACIÓN AMBIENTAL}

Para la implementación de un programa eficaz en educación ambiental se requieren lo siguientes componentes:

a) Sistematizar los conocimientos humanísticos, sociales y ciencias del medio ambiente.

b) Identificar una variedad de problemas.

c) Discernir diferentes problemas ambientales para aplicar así las soluciones correctas.

d) Promover las cualidades y aptitudes personales para superar las dificultades $y$ desarrollar las actitudes.

Para el desarrollo de las temáticas para sobre la educación ambiental la podemos determinar en cuatro etapas de acuerdo al grado de complejidad que va a depender de la edad de los usuarios receptores de la información.

Etapa 1. Teoría sobre ecología, con el fin de concebir el entorno natural que rodea al ser humano, observando sus fundamentos y funciones.

- O.G. Promover cambios de comportamientos y edificación de una conciencia socioambiental, propuesta a la adopción de nuevos valores sociales, orientados hacia el mejoramiento de las condiciones de vida de la comunidad UNESUM.

Etapa 2. Problemas Ambientales, en esta parte es importante la observación y evaluación los diferentes factores naturales y/o Antrópicos que presentan afectaciones negativas al medio.

- O.E. Estimular a la comunidad Universitaria en la temática ambiental, tomando en cuenta el reciclaje como valor fundamental de innovación y cambio de pensamiento del hombre.

Etapa 3. Evaluación de soluciones. En esta etapa se valoran la solución a las diferentes clases y características de problemas ambientales.

- O.E. Conseguir que la comunidad Universitaria comprendan sobre el valor fundamental que tiene en sus vidas el conocimiento y manejo de los desechos sólidos y la correspondencia entre este, el ambiente y su calidad de vida para alcanzar el Buen Vivir.

Etapa 4. Cooperación, en esta etapa se involucra a la comunidad en la implementación de la solución adecuada y conveniente, a los problemas ambientales, este involucra:

- Tácticas para llevar a cabo acciones individuales o colectivas.

- Toma de decisiones sobre las tácticas o alternativas que puedan seguirse.

- Valoración de resultados de las acciones iniciadas.

Cada etapa está ligada con actividades como:

- Talleres

Freddy A. Marcillo Merino, Diana V. Marcillo Parrales, Xavier E. Soledispa Rodríguez y Christian R. 
- Taller de Planificación del Programa de Educación Ambiental

- Taller de Evaluación y Planificación del Programa de Educación Ambiental en la UNESUM

- Taller de Fortalecimiento al Proyecto RECICLA - UNESUM.

- Charlas en temas ambientales

- Manejo de Desechos Sólidos

- Contaminación Ambiental

- Reciclaje

- Contacto con apoyos externos

- Ministerio de medio Ambiente

- Carrera Ingeniera en Medio Ambiente UNESUM

- Docentes Investigadores

- Eventos

- Día del Medio Ambiente

- La semana del reciclaje

- Seminarios y capacitaciones científicas

- Foros y simposios

\section{EL CONTRATO DE APRENDIZAJE}

Se realizó el análisis de la incidencia de la cultura ambiental sostenible a la comunidad de la Universitaria a través de las TI, para que no depositen basura al suelo, con el objeto de reciclar los desechos por medio de la caracterización de estrategias para atacar causas y efectos negativos que producen la Contaminación Ambiental. El desarrollo de la solución se realizó a través de un programa de educación ambiental en donde el docente y educando asumen un rol preponderante para alcanzar resultados óptimos.

Desde esta perspectiva, es de real importancia dentro del aula de clases generar espacios de debate acerca de las problemáticas medio ambientales y de sus efectos en la naturaleza, en donde el ser humano juega un papel preponderante. El cumplimiento de las etapas y las actividades de acuerdo a la formación en la rama de educación ambiental fue de real efecto, ya que se obtuvieron buenos resultados en base a un modelo de capacitaciones acordes con el entorno y la edad de los participantes. Se promovió cambios en el comportamiento y la conciencia socio-ambiental, propuesta a la adopción de nuevos valores sociales, orientados hacia el mejoramiento de las condiciones de vida de la comunidad de la Universidad Estatal del Sur de Manabí, se estimuló en la temática ambiental, tomando en cuenta el reciclaje como valor fundamental de innovación y cambio de pensamiento del hombre y se conseguió que comprendan sobre el valor fundamental 
que tiene en sus vidas el conocimiento y manejo de los desechos sólidos y la correspondencia entre este, el ambiente y su calidad de vida para alcanzar el Buen Vivir.

En conclusión, la problemática ambiental que afecta a la Universidad Estatal del Sur de Manabí, ha generado efectos negativos sobre el ambiente y los involucrados de la IES. Por lo que el ser humano debe educarse y llegar a entender que tiene que cuidar el medio ambiente e integrarse para lograr el Buen Vivir en base al respeto de los valores y derechos de la naturaleza.

\section{REFERENCIAS BIBLIOGRÁFICAS}

Adell, J. (1996). Internet en educación: una gran oportunidad. Net Conexión, 11(5).

Arnau, A. (2000). El medio ambiente: problemas y soluciones. Ediciones Miraguano.

ARROYO, VALERA S. (2004). El uso de las Nuevas Tecnologías Educativas, en la VII Conferencia de Tecnología. Universidad de Málaga, Tenerife, 3.

Cabero, J. (2010). Reflexiones sobre la brecha digital y la educación. Consejeria de Educación y Cultura, 23-42.

Cabero, j. y. (2005). Las Tic y la educación Ambiental . Revista Latinoamericana de Tecnología Educativa, 9-26.

Caicedo, Ch. Rodriguez, A. Caicedo, F. Acuña, R. Delgado, G. (2015). Plataforma virtual a través de una infraestructura basada en el sistema de gestión de aprendizajes. Sinapsis, 7(1).

Calvo, S., Corraliza, J.A. (2002). Educación ambiental. La Habana: Universidad de Pinar del Río "Hermanos Saíz Montes de Oca".

Cañal, P., Porlán, R. y García, E. (1998). Constructivismo y Enseñanza de las Ciencias. Sevilla: Díada Editora.

Cañal, P., Porlán, R. y García, E. (1981). Ecología y Escuela. Barcelona: Editorial Laia.

Castells, M. (2001). Internet y la sociedad red. Recuperado de: Lección inaugural del programa de doctorado de la Universidad Oberta de Cataluña. [en línea] Recuperado de: <http://www.uoc.es/web/esp/articles/castells/print.html/>.

Crook, C. (2009). Ordenadores y aprendizaje colaborativo. Madrid: Morata, 54.

Frosch, A. (2001). La ecología industrial del siglo XXI. Caracas (Venezuela): Editorial Ciencias.

John C. Smyth. (1996). A national strategy for environmental education: an approach to a sustainable future? The Environmentalist 16, 49-53. 
Leff, E. (2015). Aventuras de la epistemología ambiental. Ed. Siglo XXI, México, 12.

Secretaria Nacional de Planificacipon y Desarrollo. (17 de 10 de 2016). Plan Nacional del Buen Vivir. Obtenido de Responsabilidades del Plan Nacional para el Buen Vivir 2013-2017. Recuperado de: <http://www.buenvivir.gob.ec/herramientas/>.

Sigea. (5 de 3 de 2016). Las TIC y su impacto sobre el medioambiente. Recuperado de: <http://www.sigea.es/las-tic-impacto-sobre-el-medioambiente/>.

Sureda, J. (2001). Guia de la Educación Ambiental. Fuentes documentales. Conceptos Básicos. Anthropos, 54.

UNESCO. (1977). Medio Ambiente. Conferencia.

Valdiviezo, B. (2012). Los residuos tecnológicos. CEPAL, 2.

Vizcarro, C. y León, J. A. (2010). Nuevas tecnologías para el aprendizaje. Madrid: Pirámide, 35. 